IOS Press

\title{
Review
}

\section{The Rationale for Post-Operative Radiation in Localized Bladder Cancer}

\author{
Brian C. Baumann ${ }^{\mathrm{a}, \mathrm{b}}$, Paul Sargos ${ }^{\mathrm{c}}$, Libni J. Eapen ${ }^{\mathrm{d}}$, Jason A. Efstathiou ${ }^{\mathrm{e}}$, Ananya Choudhury ${ }^{\mathrm{f}, \mathrm{g}}$, \\ Amit Bahl ${ }^{\mathrm{h}}$, Vedang Murthy ${ }^{\mathrm{i}}$, Leslie K. Ballas ${ }^{\mathrm{j}}$, Valérie Fonteyne ${ }^{\mathrm{k}}$, Pierre M. Richaud ${ }^{\mathrm{c}}$, \\ Mohamed S. Zaghloul, ${ }^{1, \mathrm{~m}}$ and John P. Christodouleas ${ }^{\mathrm{a}, *}$ \\ ${ }^{a}$ Hospital of the University of Pennsylvania, Philadelphia, PA, USA \\ ${ }^{\mathrm{b}}$ Washington University in Saint Louis, Saint Louis, MO, USA \\ ${ }^{\mathrm{c}}$ Institut Bergonié, Bordeaux, France \\ ${ }^{\mathrm{d}}$ University of Ottawa, Ottawa, Canada \\ ${ }^{\mathrm{e}}$ Massachusetts General Hospital, Boston, MA, USA \\ ${ }^{\mathrm{f}}$ Department of Clinical Oncology, The Christie NHS Foundation Trust, Manchester, UK \\ ${ }^{\mathrm{g}}$ The University of Manchester, Manchester Academic Health Science Centre, Manchester, UK \\ ${ }^{\mathrm{h}}$ University Hospitals Bristol NHS Foundation Trust, Bristol, UK \\ ${ }^{\mathrm{i}}$ Tata Memorial Center, Mumbai, India \\ ${ }^{\mathrm{j}}$ University of Southern California, Los Angeles, CA, USA \\ ${ }^{\mathrm{k}}$ Ghent University Hospital, Ghent, Belgium \\ ${ }^{1}$ National Cancer Institute, Cairo University, Cairo, Egypt \\ ${ }^{\mathrm{m}}$ Children's Cancer Hospital, Cairo, Egypt
}

\begin{abstract}
Local-regional recurrence for patients with $\geq$ pT3 disease after radical cystectomy is a significant problem. Chemotherapy has not been shown to reduce the risk of local-regional recurrences in randomized prospective trials, and salvage therapies for local-regional failure are rarely successful. There is promising evidence, particularly from a recent Egyptian NCI trial, that radiation therapy plus chemotherapy can significantly reduce local recurrences compared to chemotherapy alone, and that this improvement in local-regional control may translate to meaningful improvements in disease-free and overall survival with acceptable toxicity. In light of the high rates of local failure following cystectomy for locally advanced disease and the progress that has been made in identifying patients at high risk of failure and the patterns of failure in the pelvis, the NCCN guidelines were revised in 2016 to include post-operative radiotherapy as an option to consider for patients with $\geq$ pT3 disease. Despite advances in our understanding of the problem of local-regional failure after cystectomy and the potential role of adjuvant radiotherapy, the question of whether adjuvant radiotherapy should have a defined role for patients with locally advanced urothelial carcinoma has not yet been determined. The results of the NRG, European, Indian, and Egyptian trials on adjuvant radiotherapy are eagerly awaited. While none of these trials on their own may provide definitive conclusions, their aggregate outcomes will help clarify whether this treatment should have a role in the management of patients with locally advanced bladder cancer.
\end{abstract}

Keywords: Adjuvant radiation, radical cystectomy, bladder cancer

\footnotetext{
${ }^{*}$ Correspondence to: John P. Christodouleas, MD, MPH, Department of Radiation Oncology, Hospital of the University of Pennsylvania, 3400 Civic Center Blvd, PCAM 2, Philadelphia,
}

PA 19103, USA. Tel.: +1 215662 2428; E-mail: Christojo@uphs. upenn.edu. 


\section{INTRODUCTION}

Local-regional recurrence following radical cystectomy for patients with locally advanced urothelial carcinoma is common. The risk of local-regional recurrence is not diminished with chemotherapy, and salvage treatment is rarely successful. There has recently been growing interest in adjuvant radiotherapy for bladder cancer following cystectomy. In this review article, we discuss the problem of local-regional failure after cystectomy and review the relevant literature on adjuvant radiotherapy for bladder cancer.

\section{INCIDENCE OF LOCAL-REGIONAL FAILURES}

Radical cystectomy and pelvic lymph node dissection is associated with 5-year overall survival of approximately $60 \%$ for disease confined to the bladder (pT2), but overall survival decreases to only $10-50 \%$ when disease extends into the extravesicular tissues ( $\geq$ pT3), with most patients dying with distant metastases [1]. Considerable attention has been directed to the problem of distant failure after cystectomy. Chemotherapy has been utilized in an attempt to address this problem, but randomized trials of neoadjuvant chemotherapy have demonstrated only a modest improvement in overall survival while adjuvant chemotherapy trials have had mixed results $[2,3]$. Relatively little attention has been paid to the problem of local-regional recurrence after cystectomy, probably because relatively few pelvic failures were reported in the earlier surgical literature. Several institutions reported pelvic failure rates of only 7-13\% even for patients with $\geq$ pT3 disease [4-6].

Many reports under-estimated local-regional failure rates due to a number of factors. Few studies performed routine pelvic surveillance with $\mathrm{CT}$ or MRI to detect early pelvic failures [4, 5,7], which are often asymptomatic and therefore not likely to trigger an imaging evaluation during routine follow-ups [8]. Even with routine CT surveillance, there is a significant risk of false negatives that results in systematic under-detection of pelvic disease [9]. Perhaps most importantly, most studies did not report pelvic failures unless they were the first and only site of failure without synchronous distant metastases [6, 8-12].

There is good evidence that pelvic recurrences in locally advanced bladder cancer are more common than previously suspected. In the SWOG 8710 trial of radical cystectomy with or without neoadjuvant methotrexate, vinblastine, adriamycin/doxorubicin and cisplatin (MVAC), the 5-year cumulative incidence of local-regional failure for patients with $\geq \mathrm{pT} 3$ urothelial carcinoma was 32\% [1]. The 2011 update of the MRC trial of neoadjuvant cisplatin, methotrexate and vinblastine (CMV) chemotherapy followed by cystectomy or definitive radiotherapy reported a 5-year local-regional recurrence rate of $46 \%$ and $58 \%$ for cystectomy patients with and without neoadjuvant chemotherapy, respectively [13]. A meta-analysis of randomized controlled trials with or without platinum-based chemotherapy following local therapy (usually radical cystectomy) demonstrated that $26 \%$ of patients with chemotherapy had local recurrence as a first event with or without synchronous distant failure [12]. Clinically staged patients treated at MD Anderson, most of whom received chemotherapy, had 5-year local failure rates after radical cystectomy of $29 \%$ and $44 \%$, respectively, for clinical T3b and clinical T4 disease [14, 15]. The University of Pennsylvania (PENN) experience of 442 cystectomy patients who were followed with CT or MRI every six months also demonstrated a relatively high risk of local-regional recurrence for patients with $\geq \mathrm{pT} 3$ disease with a 5 -year cumulative incidence of $28 \%$ [16]. Even in the most modern series, including the PROMETRICS 2011 cohort of prospectively followed European patients who underwent cystectomy in 2011, local-regional failure was $15-39 \%$ for $\geq \mathrm{pT} 3$ patients [17]. The problem of local-regional recurrence is not confined to Europe and North America, with similarly elevated localregional failure rates reported for $\geq \mathrm{pT} 3$ patients in a large retrospective Korean cohort [18]. Collectively, these data demonstrate that local recurrence after radical cystectomy for locally advanced disease is relatively common.

\section{RATIONALE FOR ADJUVANT RADIATION THERAPY}

Investigators have hypothesized that improved local control may lead to improved disease-free and overall survival. This hypothesis is supported by several lines of evidence. Multiple retrospective surgical series have demonstrated improved survival with more extensive nodal dissections even in the absence of nodal metastasis [19]. This finding, which is being tested in the ongoing SWOG 1011 trial randomizing patients to extensive versus standard 
lymphadenectomy, suggests that eradicating occult nodal disease may improve survival by decreasing distant as well as local failure. A similar trial from the German Urologic Oncology Group trial (AOU AB 25/02) reported an improvement in cancer-specific survival with the extended lymph node dissection $(77.5 \%$ vs. $66.2 \%)$ but the difference was not statistically significant [20]. Post-hoc analysis of pT2 patients demonstrated a statistically significant overall survival benefit for the extended dissection. There is also evidence from MD Anderson that localregional recurrence often precedes, but uncommonly follows, the appearance of distant metastases, suggesting that local failure may seed distant metastases [14]. This study also reported that local-regional recurrence was an independent prognostic variable predicting distant metastasis, a finding also reported by other investigators [21].

If local failures play a role in the subsequent development of distant disease, then adjuvant therapy is needed to enhance local control. Chemotherapy does not appear to improve pelvic control in patients with locally advanced disease. There was no reduction in local-regional failures with the addition of chemotherapy to cystectomy in the SWOG 8710 study randomizing patients to neoadjuvant MVAC chemotherapy versus no chemotherapy [22, 23] or in the MRC trial randomizing cystectomy patients to neoadjuvant CMV versus no chemotherapy [13]. The retrospective PENN experience $[1,16]$ also found no reduction in local-regional failure with the addition of neoadjuvant or adjuvant chemotherapy. The inability of systemic therapy to enhance local control suggests a potential role for adjuvant local treatments such as radiation therapy to diminish local failures, and adjuvant radiation therapy is already the standardof-care for locally advanced disease for a variety of cancers, including glioblastoma multiforme, head and neck cancer, non-small cell lung cancer, breast cancer, gynecologic cancers, and soft tissue sarcoma.

The need for adjuvant therapy to reduce local failures would be less compelling if successful salvage therapies were available, as is the case following radical prostatectomy in which early salvage radiotherapy after biochemical recurrence is a viable alternative to immediate adjuvant radiation in highrisk prostate cancer patients. Unfortunately, salvage treatment of clinically detected local-regional recurrences after cystectomy is rarely successful, with a median survival of 9 months following development of local-regional recurrence [24]. In the PENN series, only 1 in 80 patients with pelvic recurrence remained alive at 5 years [24]. The large doses of radiation necessary to control grossly recurrent bladder cancer is usually precluded by the close proximity of critical normal structures, particularly bowel and the patient's neobladder/urinary diversion [25]. Even if there is no discernible improvement in survival from adjuvant local therapy, there is a rationale for adjuvant local therapy to prevent the often substantial morbidity of pelvic recurrences [26] which can cause pain and ureteric, venous, or lymphatic obstruction.

\section{EFFICACY OF ADJUVANT RADIATION THERAPY IN THE EXISTING LITERATURE}

More direct support for adjuvant radiation therapy is provided by promising outcomes in small prospective studies in Egyptian cohorts and an Italian study. Zaghloul et al. (1992) conducted a trial of post-operative radiotherapy that randomized 236 radical cystectomy patients with pT3a-pT4a disease to receive either observation or post-operative radiotherapy given either as $37.5 \mathrm{~Gy}$ in $1.25 \mathrm{~Gy}$ fractions given three times per day separated by at least 3 hours over 12 days OR 50 Gy in 2 Gy daily fractions over 5 weeks [27]. Patients were enrolled from $1981-1984$. The study reported 5 year DFS of $44 \%$ with conventional fractionation, $49 \%$ with hyperfractionation, and $25 \%$ with observation $(p<0.01)$. Local-regional control at 5 years also significantly favored the radiotherapy arms, with pelvic control rates of $93 \%, 87 \%$, and $50 \%$, respectively $(p<0.01)$. While $80 \%$ of the patients on the study had squamous cell histology and only $20 \%$ urothelial neoplasms, the authors reported that the results of the study did not vary based on histology [27]. This trial helped to establish adjuvant radiotherapy as the standard-ofcare for locally advanced disease in Egypt, but the study did not change practice in the rest of the world where the overwhelming majority of patients have urothelial carcinoma. Post-operative radiation therapy was also an independent predictor of improved cancer-free survival in a small Italian study published in abstract form [28].

At the NCI in Egypt, post-operative radiation therapy was then tested against pre-operative radiotherapy in a randomized trial of 100 patients enrolled from 2004-2007. Both arms were treated with 2-D radiotherapy to $50 \mathrm{~Gy}$ in 25 fractions over 5 weeks [29]. There was no significant difference between the 
two arms with respect to oncologic outcomes. The study is useful for demonstrating that post-operative radiotherapy, even with the use of 2-D radiotherapy techniques, was associated with relatively modest late grade 3 GI toxicity (2 out of 50 patients).

Zaghloul et al. at the NCI in Egypt then conducted a follow-up randomized trial of adjuvant radiation therapy that compared radiation vs. sequential chemo+RT after radical cystectomy for locally advanced bladder cancer. A third arm was later added using adjuvant chemotherapy alone. The results for the randomized phase II comparison of the sequential chemotherapy plus RT vs. chemotherapy alone arms were presented at ASTRO 2016 [30]. The trial included locally advanced bladder cancer patients ( $\geq$ pT $3 b$, grade 3 , or positive nodes) who had negative margins after surgery. Chemo+RT included 2 cycles of gemcitabine/cisplatin before and after RT to $45 \mathrm{~Gy}$ in $1.5 \mathrm{~Gy}$ twice-daily fractions using $3 \mathrm{D}$-conformal techniques. Chemotherapy alone included 4 cycles of gemcitabine/cisplatin. The chemo+RT arm accrued 75 patients from $2002-2008$. The chemotherapy alone arm accrued 45 patients from 2007-2008 with a $4: 1: 1$ randomization weighted toward chemotherapy alone. The primary endpoint was local-regional recurrence free survival (LRFS). Fifty-three percent had urothelial carcinoma; $47 \%$ had squamous cell carcinoma/other. Median follow-up was 21 and 17 months for the chemo+RT and chemotherapy arms, respectively. The two arms were balanced except for age (mean 52 vs. 55 years, $p=0.04$ ) \& tumor size (mean 5 vs. $6 \mathrm{~cm}, p<0.01$ ), both favoring chemo+RT. Two-year outcomes and overall adjusted hazard ratios (HR) for chemo+RT vs. chemo alone were 96\% vs. $69 \%$; HR 0.08 (95\% CI $0.02-0.39, p<0.01)$ for LRFS, $68 \%$ vs. $56 \%$; HR 0.53 (95\% CI $0.27-1.06$, $p=0.07$ ) for disease-free survival and $71 \%$ vs. $60 \%$; HR 0.61 (95\% CI $0.33-1.11, p=0.11$ ) for overall survival. The study demonstrated a significant improvement in local-regional recurrence and a marginally significant improvement in disease-free survival and overall survival with the addition of radiotherapy. RTassociated late grade 3+ GI toxicity was observed in 5 patients $(7 \%)$ in the chemo+RT arm.

This study reports promising outcomes but has important limitations. The weighted randomization when the 3rd arm was added later caused imbalances between the chemotherapy alone and chemo+RT arms in terms of age and tumor size. The relatively small size of the patient cohort $(n=120)$ may be underpowered to detect a statistically significant difference in survival between the two arms. The heterogeneity of the tumor histology, with a sizeable minority of patients having non-urothelial disease, may limit the applicability of the study to patient populations outside of the Middle East.

\section{TREATMENT-RELATED TOXICITIES OF ADJUVANT RADIATION}

Although early studies demonstrated that adjuvant radiotherapy decreased pelvic recurrences and in some cases improved disease-free survival and even overall survival compared to cystectomy alone, interest in adjuvant radiotherapy waned decades ago, in large measure because of excessive gastrointestinal toxicity associated with older radiation techniques from the 1970s-1980s and improvements in surgical technique which many investigators believed obviated the need for adjuvant radiation [31, 32]. The clinical trial of post-operative radiation vs. observation conducted by Zaghloul et al. at the NCI in Egypt between 1981-1984 reported significant late GI toxicity in 14 of the 39 assessable patients (36\%) treated with standard fractionation although late GI toxicity was reduced in the group treated with accelerated hyperfractionation (125 cGy fractions) with significant late GI toxicity in 4 of 51 patients $(10 \%)$ [27]. Reisinger et al. reported severe late toxicity in 20 of 40 patients $(50 \%)$ treated with sandwich radiotherapy [31]. A study by Spera et al. reported that $59 \%$ of the 22 patients treated with $5 \mathrm{~Gy} \times 1$ pre-operative radiation therapy followed by $45 \mathrm{~Gy}$ in 4.5 weeks of post-operative radiation had significant post-treatment complications [28]. The high reported toxicity for adjuvant radiation was likely due to a combination of radiation-specific factors as well as the high rates of toxicity seen with cystectomy alone in early series. Radiation specific factors included the use of older 2-D radiotherapy techniques, large radiation treatment fields encompassing the entire pelvis, and field arrangements using AP/PA beams which would increase bowel dose compared to other field arrangements [31]. The high complication rates of cystectomy alone were demonstrated in the Spera series, in which 10 of 20 cystectomy patients $(50 \%)$ had serious complications compared to $59 \%$ in the cystectomy plus RT cohort. Larger cystectomy series have demonstrated similar findings. In the Mayo Clinic experience of 1,057 cystectomy patients who underwent urinary conduit diversion from 1980-1998, significant conduit-related complications occurred in $61 \%$ of patients, with $20 \%$ 
having bowel complications and 16\% having stomal complications [33]. Complication rates after surgery alone are significant even in more modern series. The German AUO trial of standard vs. extended lymph node dissection reported 90 day Clavien grade $\geq 3$ complications of 26-30\% and 90 day post-operative mortality of $4 \%$. The studies of adjuvant radiation therapy in the USA with older radiation and surgical techniques were small and toxicities were high, resulting in abandonment of adjuvant radiation, although it could be argued that the marginal toxicity of adjuvant radiation was relatively low in comparison to the toxicity of cystectomy alone. The late grade $3+$ GI toxicity of adjuvant chemo-radiotherapy using modern 3-D conformal radiotherapy in the Egyptian trial reported at ASTRO 2016 was only 7\%, suggesting that adjuvant radiotherapy with modern techniques can be delivered with considerably less toxicity than what was reported in the past.

\section{SELECTING PATIENTS FOR ADJUVANT RADIATION TRIALS}

A critical challenge for modern trials of adjuvant radiotherapy is the need to select patients appropriately who have a high risk for local-regional failure and are therefore more likely to benefit from adjuvant local therapy. Prior studies to identify the risk factors predicting local failure produced conflicting results. Myriad factors were reported to predict pelvic relapse on univariate analysis, including: $\mathrm{pT}$ stage $[1$, 14, 23], pathologic nodal status [21, 23, 34], mixed histology disease [35], surgical margins $[1,7,15$, $23]$, extent of node dissection [1, 16, 35], density of positive nodes [36], and patient age [23, 34]. Investigators at PENN developed a risk stratification model to identify patients at increased risk of local-regional recurrence based on a cohort of 442 consecutive cystectomy patients treated from 1990-2008, 29\% of whom received adjunctive chemotherapy, and all of whom were followed with routine pelvic CT/MRI following surgery [16]. Local-regional failure was defined as any failure below the aortic bifurcation detected before or within three months of distant metastases, excluding the inguinal nodes. On multivariate competing risk analysis, $\geq \mathrm{pT} 3$ stage and number of nodes excised ( $<10$ vs. $\geq 10)$ were significant independent predictors of local failure. Pelvic nodal involvement was a predictor of local-regional failure on univariate analysis but was not predictive on multivariate analysis, which may be due to the relatively high competing risk of distant disease for patients with involved nodes.

For validation, the PENN experience was compared with data from the SWOG 8710 study of 317 patients with cT2-T4a disease randomized from 1987 to 1998 to receive cystectomy with or without neoadjuvant MVAC chemotherapy (19). From the combined cohorts, a risk stratification model was developed using pathologic stage, number of nodes excised, and margin status. Three patient subgroups were identified with significantly different local failure risk: low-risk $(\leq \mathrm{pT} 2)$, intermediate-risk $(\geq \mathrm{pT} 3$ with negative margins and $\geq 10$ nodes identified), and high-risk ( $\geq$ pT3 with positive margins $\mathrm{OR}<10$ nodes identified) with 5-year cumulative incidence of LF of $8 \%, 20 \%$, and $41 \%$ in the SWOG cohort and $8 \%, 19 \%$, and $41 \%$ in the PENN cohort (1) (Fig. 1). The risk stratification model identified subgroups that have significantly different overall survival, confirming its clinical relevance. The subgroups did not have significantly different rates of isolated distant metastasis, suggesting that the model is not merely a predictor of distant disease. This model was externally validated in two additional patient cohorts. Froehner et al. validated the model using a multinational, European prospective cohort of 565 patients (PROMETRICS 2011) [17, 37]. Ku et al. also validated the model in a retrospective cohort of 398 Korean patients, suggesting that the risk stratification is valid not only in a US or European population but also in a large Asian cohort [18].

To address concerns that a risk stratification model developed using historical databases may not accurately reflect the patterns and incidence of failures associated with contemporary surgical practice, the year of cystectomy was used as a proxy for the

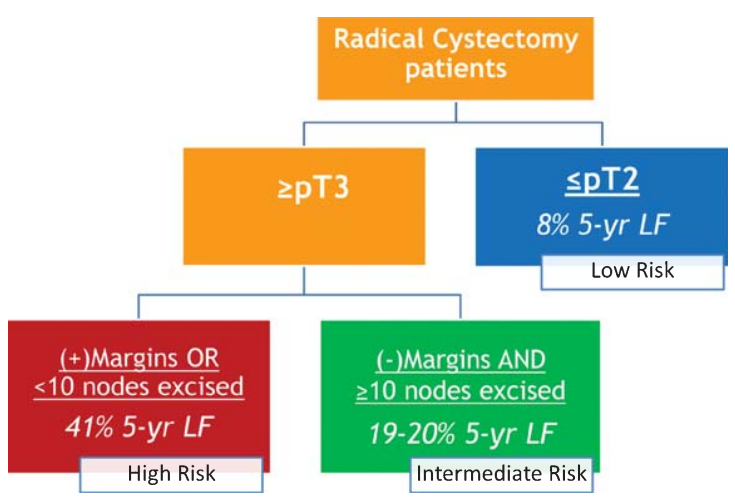

Fig. 1. PENN risk stratification for predicting local-regional recurrence after radical cystectomy. 
evolution of surgical techniques over time, and no differences were found in the local failure estimates for each subgroup as a function of the year of surgery [38]. This finding lends credence to the validity of the model to identify patients at increased risk for localregional failure in the modern era, even though the model was developed from a cohort in which many of the patients were operated on in the late 1980s and 1990's. Additional support for this conclusion can be drawn from the fact that the model was externally validated by Froehner et al. whose patient database was limited to patients undergoing cystectomy in 2011 [37].

The PENN model was originally developed using a cohort of consecutive patients from a single institution, so there was concern that the model may not accurately predict the expected local-regional failure rates that would be observed in a protocol setting in which trial accrual bias might result from the exclusion of patients who develop local failure, distant metastases, are lost to follow-up, or die soon after their cystectomy before they could be enrolled in a study of adjuvant therapy [38]. To assess the potential impact of trial accrual bias on the model, the investigators determined the model's ability to stratify patients into distinct risk groups when patients likely to be excluded from a prospective protocol were censored from the patient databases. They found that trial accrual bias would not affect the risk stratification model's validity and would not be likely to change the model's predictions about the absolute rate of local-regional recurrence associated with each risk category [38].

Additional analysis also shed light on the effect of different subgroup definitions on the results of the PENN risk stratification. In particular, the authors evaluated several of the assumptions used in the NRG-GU001 trial randomizing patients at intermediate or high-risk of local failure after radical cystectomy with or without chemotherapy to either observation or adjuvant radiation therapy to $50.4 \mathrm{~Gy}$. The NRG trial adopted the PENN externally validated risk stratification model with the exception that the trial designers excluded patients from their intermediate risk group who had pT3a disease with negative margins and more extensive nodal dissections. In the initial analysis of the PENN cohort, the authors investigated whether to group these relatively more favorable pT3a patients with the low-risk or intermediate-risk subgroups but found that the Harrell's c-index was maximized if these patients were grouped with the intermediate-risk cohort [16], but it was clear that these pT3a patients had more favorable local failure rates compared to other patients in the intermediate-risk subgroup. When the NRG criteria were applied to the PENN-SWOG cohorts, the two risk groups defined by the NRG risk stratification (intermediate and high-risk) have significantly different LF rates, supporting the validity of the risk stratification model [38]. In addition, the 35 pT3a patients who were excluded from the intermediaterisk group had significantly lower rates of LF than either the NRG intermediate risk group or the NRG high risk group, further supporting the validity of the NRG's modification of the PENN risk model [38].

Reddy et al. recently proposed an alternative to the PENN risk stratification [39]. In their single institution series, only $\mathrm{pT}$ stage and $\mathrm{pN}$ stage were significant independent predictors of pelvic failure. There is conflicting evidence in the literature about the importance of $\mathrm{pN}$ stage as a predictor of pelvic recurrence, possibly related to differences in statistical methodology. For example, the PENN risk stratification was developed using cumulative incidence and competing risk analysis with death, isolated distant metastases, and second malignancies treated as competing events [1], the Reddy study used standard Kaplan-Meier survival analysis and Cox regressions. In the setting of locally advanced bladder cancer where the risk of competing events is high, standard survival analysis generates higher estimates for pelvic failure than cumulative incidence methods, which can affect the risk factor analysis [40]. In a previous analysis of the PENN data, $\mathrm{pN}$ stage was a significant independent predictor of pelvic failure using Cox regression analysis but was not a significant independent predictor using the competing risk approach [40]. Additional analysis is needed to study the effect of $\mathrm{pN}$ stage on rates of pelvic failure.

\section{LOCAL-REGIONAL FAILURE BY PELVIC SUB-REGIONS}

It is insufficient merely to identify patients at elevated risk of pelvic failure. The patterns of failure within the pelvis must also be defined to identify appropriate radiation therapy targets and to predict the toxicity of treating such targets. Two single institutional series have addressed this issue for the purposes of rationalizing adjuvant radiation therapy target volumes. The study by Baumann et al. reviewed 
(A)

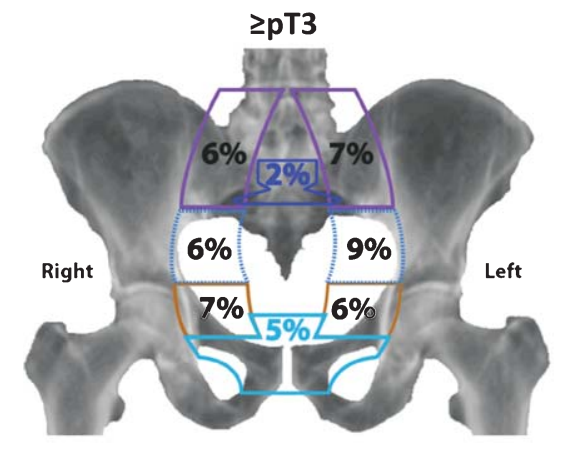

(B)

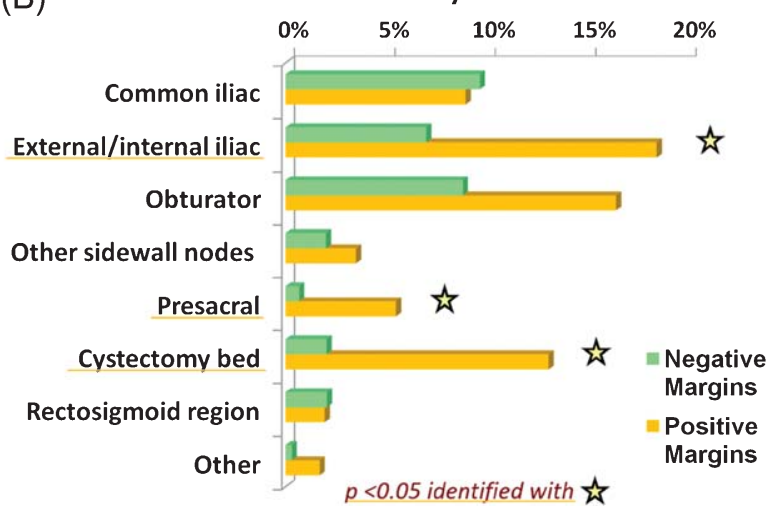

Fig. 2. (A) Five year cumulative incidence of local-regional failure by location of recurrence for stage $\geq$ pT3 patients. Local-regional failures were defined as recurrences in the pelvic lymph nodes or soft tissues before or within 3 months of evidence of distant failure. The pelvic sidewall nodes are the common iliac, external/internal iliac, and obturator nodes (from top to bottom). The structures in the middle of the pelvis are the presacral nodal region (superiorly) and the cystectomy bed. (B) Five year cumulative incidence of local-regional failure by site for $\geq$ pT3 patients with positive vs. negative surgical margins.

5-year LF rates after cystectomy for 8 pelvic sites among 442 urothelial bladder carcinoma patients [24]. Patients with $\geq$ pT3 disease had significantly higher LF rates at nearly all pelvic sites compared to early-stage patients (Fig. 2A). Among stage $\geq$ pT3 patients, margin status significantly altered the pattern of failure, but extent of node dissection and nodal positivity did not. In stage $\geq \mathrm{pT} 3$ patients with negative margins, failures occurred primarily in the common iliac, internal/external iliac, and obturator nodes with 5-year LF rates of $9.7 \%, 7.0 \%$, and $8.8 \%$, respectively, while failure in the cystectomy bed and/or presacral nodes was uncommon (5-year LF rates of $2 \%$ and $0.7 \%$, respectively) (Fig. 2B). For these patients, $76 \%$ of all their subsequent sites of local failure would be encompassed within CTVs covering only the iliac/obturator nodes. For stage $\geq \mathrm{pT} 3$ patients with positive margins, failures were still predominantly in the pelvic sidewall nodal region, with 5 year LF rates of $9 \%, 18.5 \%$, and $16.5 \%$ for the common iliac, external/internal iliac, and obturator nodes, but the incidence of cystectomy bed and presacral nodal failures increased significantly (13.1\% and 5.5\%, respectively) (Fig. 2B). Clinical target volumes limited to the iliac/obturator nodal regions would only encompass all sites of failure in $57 \%$ of patients with positive margins destined to develop pelvic recurrence. Including the presacral and cystectomy bed regions increased the percentage of local failures encompassed within the radiation portals to $91 \%$. This data suggested that adjuvant RT protocols should target at least the obturator and iliac regions in stage $\geq$ pT3 patients with negative margins. Coverage of the presacral region and cystectomy bed may be warranted for stage $\geq \mathrm{pT} 3$ patients with positive surgical margins [24]. The study of pelvic failure by Baumann et al. was based on a single-institution's experience and has not been externally validated. The study by Reddy et al. also showed that most failures occurred in the pelvic sidewall nodal regions with an increase in failures noted in the cystectomy bed and presacral region when the surgical margins were positive [39]. For pT3 and pT4 patients, 2 year LF rates were $8 \%$ and $11 \%$ for the common iliac nodal region, $6 \%$ and $15 \%$ for the external/internal iliac region, $7 \%$ and $19 \%$ for the obturator nodal region, $4 \%$ and $6 \%$ for the cystectomy bed, respectively. The authors found that there was a significantly increased risk of failure in the pelvic sidewall nodal regions for patients who had pathologically involved nodes at the time of surgery, an inconsistency with the prior patterns of failure analysis that may be related to differences in the way competing risks were handled, as mentioned previously.

\section{ADJUVANT RADIATION PLANNING AND DELIVERY TECHNIQUES}

Early studies of adjuvant radiotherapy in the US used 2-D radiation treatment techniques and were associated with relatively high rates of late GI toxicity using pre-1980s radiation techniques [31]. A more precise identification of the organs at risk, combined with advances in radiation delivery modalities, such 
as 3-D conformal radiotherapy, intensity modulated radiation therapy (IMRT), and proton therapy, could potentially reduce GI toxicity and improve the therapeutic ratio of adjuvant radiotherapy. The only study to report a comparison of different treatment techniques assessed the effect on bowel and rectal dose of three different treatment modalities treating clinical target volumes that encompassed either the pelvic sidewall nodal region alone (for negative margin patients) or the pelvic sidewall nodal region plus the presacral region and the cystectomy bed (for positive margin patients) [41]. The study reported a dosimetric advantage for IMRT and pencil beam scanned proton radiotherapy over 3-D conformal radiation to improve rectal and bowel dose, although the clinical importance of such results could only be confirmed on a clinical trial. There was no significant difference between the proton and IMRT plans with respect to bowel or rectal dose. Based on this dosimetric analysis, the trials at the NRG, GETUG-AFU, the University of Ghent, and Tata Memorial Hospital in India require an IMRT approach.

With the adoption of highly conformal radiation delivery techniques comes a greater emphasis on careful delineation of the clinical target volumes to prevent a marginal miss. International consensus guidelines were developed for the clinical target volumes and organs at risk for adjuvant radiation therapy, as these structures were not well-defined in the literature [25]. The purpose of this study was to achieve a multi-disciplinary, multi-institutional, international consensus defining clinical target volumes (CTVs) and organs-at-risk (OARs) for bladder cancer patients undergoing adjuvant radiation in clinical trials and to describe those targets and OARs in a way that generates consistent contours. Twenty-five radiation oncologists and urologists from 16 institutions and 5 countries participated in the study. The group proposed that patients at elevated risk for local-regional failure with negative margins should be treated to the pelvic nodes alone (internal/external iliac, distal common iliac, and presacral) whereas patients with positive margins should be treated to the pelvic nodes and cystectomy bed [24, 25]. Proposed OARs included the rectum, bowel space, bone marrow, and urinary diversion. The area of greatest discussion in the development of the consensus contours was the cystectomy bed CTV. Consensus language describing the CTVs and OARs was developed and externally validated, and the revised instructions were found to produce consistent contours [25]. The consensus contouring guidelines have been adopted in the ongoing trials at the NRG, GETUG-AFU, Tata Memorial Hospital, and the University of Ghent.

\section{ONGOING AND PROPOSED PROSPECTIVE STUDIES OF ADJUVANT RADIATION}

Multiple clinical trials of post-operative radiotherapy have recently opened or are in development around the world, including three cooperative group trials the currently accruing NRG trial (NRGGU001), the GETUG-AFU trial in France, and the proposed NCRI trial in the UK as well as single institution trials at Tata Memorial Hospital in India, the University of Ghent in Belgium, and the NCI in Cairo [40, 42]. Table 1 summarizes design elements of these trials. Future efforts may examine the role of adjuvant radiotherapy in combination with immunotherapy and chemotherapy, as there may be a synergistic effect with radiation and certain immune therapies (e.g. anti-PD-1 and anti-PD-L1 inhibitors).

\section{CONCLUSIONS}

Local regional recurrence for patients with $\geq \mathrm{pT} 3$ disease after radical cystectomy is a significant problem. Chemotherapy has not been shown to reduce the risk of local-regional recurrences in randomized prospective trials, and salvage therapies for local-regional failure are rarely successful. There is promising evidence, particularly from a recent Egyptian NCI trial, that radiation therapy plus chemotherapy can significantly reduce local recurrences compared to chemotherapy alone, and that this improvement in local-regional control may translate to meaningful improvements in disease-free and overall survival with acceptable toxicity. In light of the high rates of local failure following cystectomy for locally advanced disease and the progress that has been made in identifying patients at high risk of failure and the patterns of failure in the pelvis, the NCCN guidelines were revised in 2016 to include post-operative radiotherapy as an option to consider for patients with $\geq \mathrm{pT} 3$ disease [43]. Despite advances in our understanding of the problem of local-regional failure after cystectomy and the potential role of adjuvant radiotherapy, the question of whether adjuvant radiotherapy should have a defined role for patients with locally advanced urothelial carcinoma has not yet been determined. The results of the NRG, European, Indian, and Egyptian trials on adjuvant 


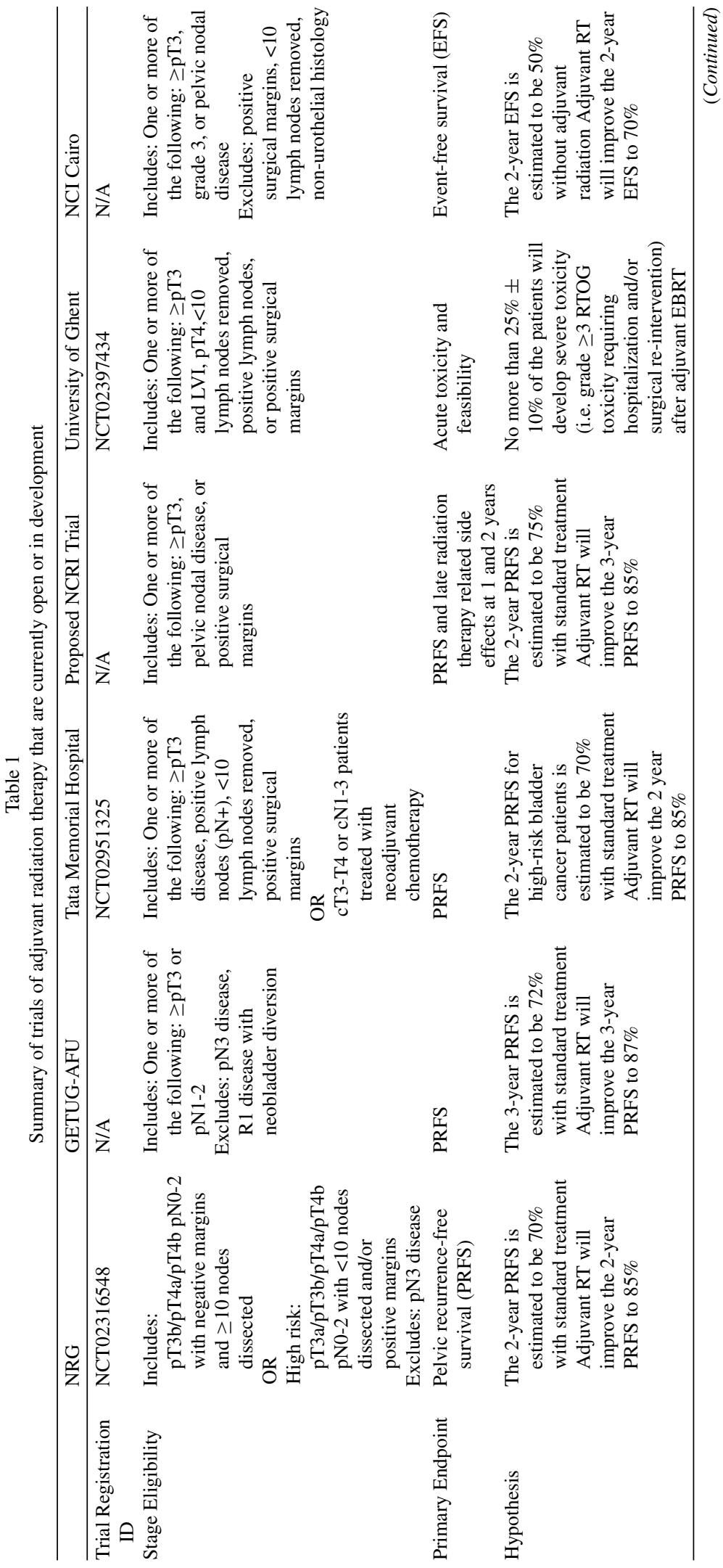




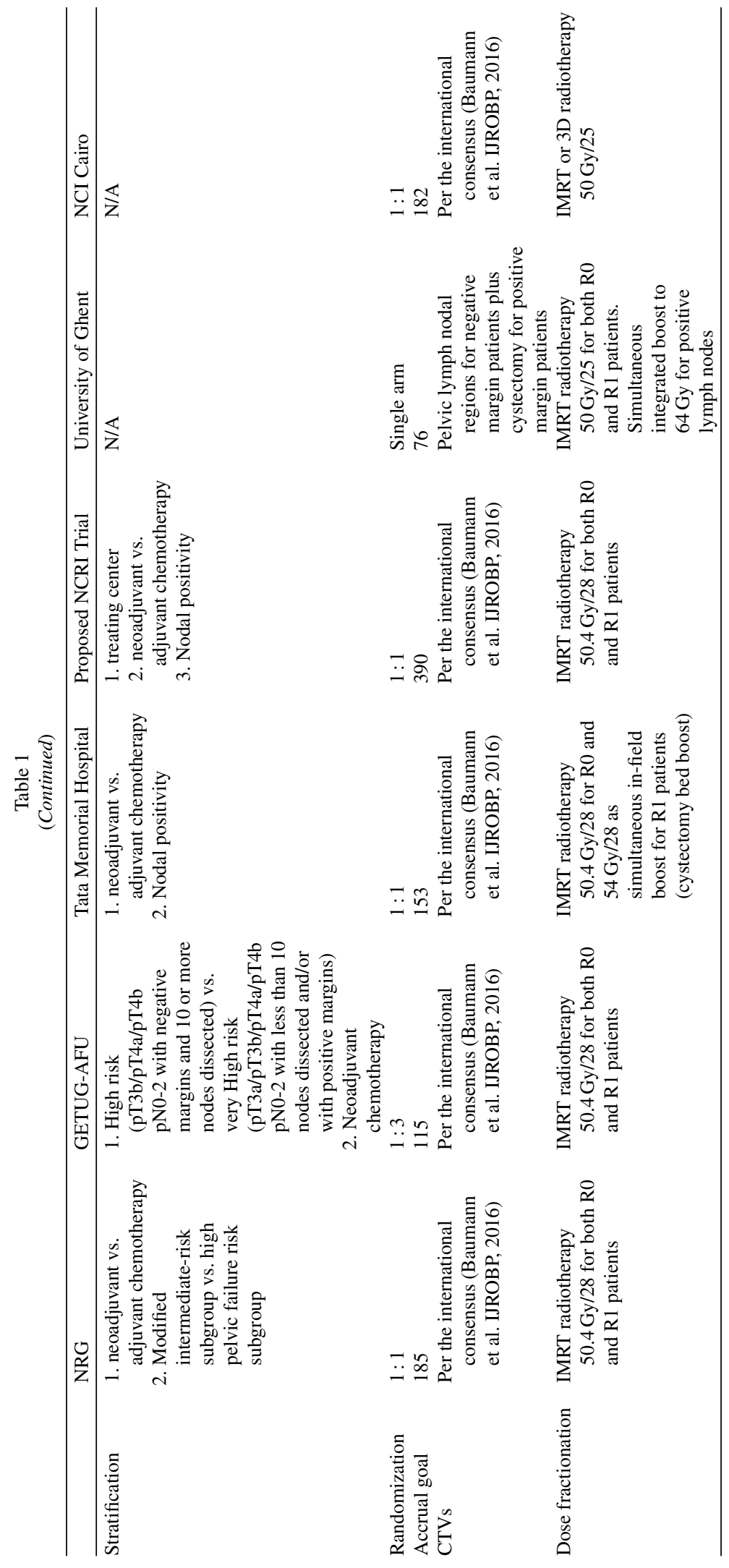


radiotherapy are eagerly awaited. While none of these trials on their own may provide definitive conclusions, their aggregate outcomes will help clarify whether this treatment should have a role in the management of patients with locally advanced bladder cancer.

\section{CONFLICT OF INTEREST}

J.C. discloses employment at Elekta AB. The authors have nothing else to disclose.

\section{REFERENCES}

[1] Christodouleas JP, Baumann BC, He J, et al. Optimizing bladder cancer locoregional failure risk stratification after radical cystectomy using SWOG 8710. Cancer 2014;120:1272-80.

[2] Kamat AM, Hahn NM, Efstathiou JA, et al. Bladder cancer. Lancet 2016;388:2796-2810.

[3] Sternberg CN, Skoneczna I, Kerst JM, et al. Immediate versus deferred chemotherapy after radical cystectomy in patients with pT3-pT4 or N+ M0 urothelial carcinoma of the bladder (EORTC 30994): An intergroup, open-label, randomised phase 3 trial. Lancet Oncol 2015;16:76-86.

[4] Frazier HA, Robertson JE, Dodge RK, Paulson DF. The value of pathologic factors in predicting cancer-specific survival among patients treated with radical cystectomy for transitional cell carcinoma of the bladder and prostate. Cancer 1993;71:3993-4001.

[5] Zehnder P, Studer UE, Skinner EC, et al. Super extended versus extended pelvic lymph node dissection in patients undergoing radical cystectomy for bladder cancer: A comparative study. J Urol 2011;186:1261-8.

[6] Stein JP, Lieskovsky G, Cote R, et al. Radical cystectomy in the treatment of invasive bladder cancer: Long-term results in 1,054 patients. J Clin Oncol 2001;19:666-75.

[7] Visser O, Nieuwenhuijzen JA, Horenblas S. Local recurrence after cystectomy and survival of patients with bladder cancer: A population based study in greater amsterdam. J Urol 2005; 174:97-102.

[8] Hassan JM, Cookson MS, Smith JA Jr, Chang SS. Patterns of initial transitional cell recurrence in patients after cystectomy. J Urol 2006;175:2054-7.

[9] Volkmer BG, Kuefer R, Bartsch GC Jr, et al. Oncological followup after radical cystectomy for bladder cancer-is there any benefit? J Urol 2009;181:1587-93.

[10] Madersbacher S, Hochreiter W, Burkhard F, et al. Radical cystectomy for bladder cancer today-a homogeneous series without neoadjuvant therapy. J Clin Oncol 2003;21:690-6.

[11] Millikan R, Dinney C, Swanson D, et al. Integrated therapy for locally advanced bladder cancer: Final report of a randomized trial of cystectomy plus adjuvant M-VAC versus cystectomy with both preoperative and postoperative M-VAC. J Clin Oncol 2001;19:4005-13.

[12] Neoadjuvant chemotherapy for invasive bladder cancer. Cochrane Database Syst Rev 2005:CD005246.

[13] International Collaboration of T, Medical Research Council Advanced Bladder Cancer Working P, European Organisation for R, et al. International phase III trial assessing neoadjuvant cisplatin, methotrexate, and vinblastine chemotherapy for muscle-invasive bladder cancer: Longterm results of the BA06 30894 trial. J Clin Oncol 2011;29:2171-7.

[14] Pollack A, Zagars GK, Cole CJ, et al. The relationship of local control to distant metastasis in muscle invasive bladder cancer. J Urol 1995;154:2059-63.

[15] Cole CJ, Pollack A, Zagars GK, et al. Local control of muscle-invasive bladder cancer: Preoperative radiotherapy and cystectomy versus cystectomy alone. Int J Radiat Oncol Biol Phys 1995;32:331-40.

[16] Baumann BC, Guzzo TJ, He J, et al. A novel risk stratification to predict local-regional failures in urothelial carcinoma of the bladder after radical cystectomy. Int J Radiat Oncol Biol Phys 2013;85:81-8.

[17] Novotny V, Froehner M, May M, et al. Risk stratification for locoregional recurrence after radical cystectomy for urothelial carcinoma of the bladder. World J Urol 2015;33:1753-61.

[18] Ku JH, Kim M, Jeong CW, et al. Risk prediction models of locoregional failure after radical cystectomy for urothelial carcinoma: External validation in a cohort of korean patients. Int J Radiat Oncol Biol Phys 2014;89: 1032-7.

[19] Skinner EC, Stein JP, Skinner DG. Surgical benchmarks for the treatment of invasive bladder cancer. Urol Oncol 2007;25:66-71.

[20] Gschwend JE, Heck MM, Lehmann J, Ruebben H. Limited versus extended pelvic lymphadenectomy in patients with bladder cancer undergoing radical cystectomy: Survival results from a prospective, randomized trial (LEA AUO AB 25/02) [Abstract]. J Clin Oncol 2016;34:abstr 4503.

[21] Ide H, Kikuchi E, Miyajima A, et al. The predictors of local recurrence after radical cystectomy in patients with invasive bladder cancer. Jpn J Clin Oncol 2008;38:360-4.

[22] Grossman HB, Natale RB, Tangen CM, et al. Neoadjuvant chemotherapy plus cystectomy compared with cystectomy alone for locally advanced bladder cancer. N Engl J Med 2003;349:859-66.

[23] Herr HW, Faulkner JR, Grossman HB, et al. Surgical factors influence bladder cancer outcomes: A cooperative group report. J Clin Oncol 2004;22:2781-9.

[24] Baumann BC, Guzzo TJ, He J, et al. Bladder cancer patterns of pelvic failure: Implications for adjuvant radiation therapy. Int J Radiat Oncol Biol Phys 2013;85:363-9.

[25] Baumann BC, Bosch WR, Bahl A, et al. Development and validation of consensus contouring guidelines for adjuvant radiation therapy for bladder cancer after radical cystectomy. Int J Radiat Oncol Biol Phys 2016;96:78-86.

[26] Eapen LJ, Jones E, Kassouf W, et al. Enumerating pelvic recurrence following radical cystectomy for bladder cancer: A Canadian multi-institutional study. Can Urol Assoc J 2016;10:90-4.

[27] Zaghloul MS, Awwad HK, Akoush HH, et al. Postoperative radiotherapy of carcinoma in bilharzial bladder: Improved disease free survival through improving local control. Int J Radiat Oncol Biol Phys 1992;23:511-7.

[28] Cozzarini C, Pellegrini D, Fallini M, et al. Reappraisal of the role of adjuvant radiotherapy in muscle-invasive transitional cell carcinoma of the bladder [Abstract]. Int J Radiat Oncol Biol Phys 1999;45:221-2.

[29] El-Monim HA, El-Baradie MM, Younis A, et al. A prospective randomized trial for postoperative vs. preoperative adjuvant radiotherapy for muscle-invasive bladder cancer. Urol Oncol 2013;31:359-65. 
[30] Zaghloul MS, Christodouleas JP, Smith A, et al. Adjuvant sandwich chemotherapy and radiation versus adjuvant chemotherapy alone for locally advanced bladder cancer. Int J Radiat Oncol Biol Phys 2016;96:S94.

[31] Reisinger SA, Mohiuddin M, Mulholland SG. Combined pre- and postoperative adjuvant radiation therapy for bladder cancer-a ten year experience. Int J Radiat Oncol Biol Phys 1992;24:463-8.

[32] Spera JA, Whittington R, Littman P, et al. A comparison of preoperative radiotherapy regimens for bladder carcinoma. The University of Pennsylvania experience. Cancer 1988;61:255-62.

[33] Shimko MS, Tollefson MK, Umbreit EC, et al. Longterm complications of conduit urinary diversion. J Urol 2011;185:562-7.

[34] Cheng L, Weaver AL, Leibovich BC, et al. Predicting the survival of bladder carcinoma patients treated with radical cystectomy. Cancer 2000;88:2326-32.

[35] Honma I, Masumori N, Sato E, et al. Local recurrence after radical cystectomy for invasive bladder cancer: An analysis of predictive factors. Urology 2004;64:744-8.

[36] Kassouf W, Agarwal PK, Herr HW, et al. Lymph node density is superior to TNM nodal status in predicting disease-specific survival after radical cystectomy for bladder cancer: Analysis of pooled data from MDACC and MSKCC. J Clin Oncol 2008;26:121-6.
[37] Froehner M, Novotny V, Wirth MP, et al. External validation of a model to predict locoregional failure after radical cystectomy. Cancer 2014;120:3584.

[38] Baumann BC, He J, Hwang WT, et al. Validating a local failure risk stratification for use in prospective studies of adjuvant radiation therapy for bladder cancer. Int $\mathrm{J}$ Radiat Oncol Biol Phys 2016;95:703-6.

[39] Reddy AV, Pariser JJ, Pearce SM, et al. Patterns of failure after radical cystectomy for pT3-4 bladder cancer: Implications for adjuvant radiation therapy. Int J Radiat Oncol Biol Phys 2016;94:1031-9.

[40] Christodouleas JP, Hwang WT, Baumann BC. Adjuvant radiation for locally advanced bladder cancer? A question worth asking. Int J Radiat Oncol Biol Phys 2016;94:1040-2.

[41] Baumann BC, Noa K, Wileyto EP, et al. Adjuvant radiation therapy for bladder cancer: A dosimetric comparison of techniques. Med Dosim 2015;40:372-7.

[42] Sargos P, Baumann BC, Eapen LJ, et al. Adjuvant radiotherapy for pathological high-risk muscle invasive bladder cancer: Time to reconsider? Transl Androl Urol 2016;5:70210.

[43] National Comprehensive Cancer Network (NCCN) Clinical Practice Guidelines in Oncology (NCCN Guidelines): Bladder Cancer. Version 2.2016. www.nccn.org (Accessed on October 12016 ). 